\title{
Integrated Plasmonic Nanoantenna Gratings for Large Area Coherent Optical Source
}

\author{
Benjamin Leroy ${ }^{1}$, Giovanni Magno ${ }^{1}$, David Barat ${ }^{2}$, Laetitia Pradere ${ }^{2}$, Béatrice Dagens ${ }^{1}$ \\ 1. Centre de Nanosciences et de Nanotechnologies, CNRS, Univ. Paris-Sud, Université Paris-Saclay, C2N, F-91120 Palaiseau, France \\ 2. Groupe PSA, Direction Scientifique, Centre technique de Vélizy, route de Gisy, 78140 Vélizy-Villacoublay, France
}

The emission of coherent light from a large area with a controlled directivity is required for high quality holographic system. In application domains like automotive, such a source needs to be fully planar for compactness and portability. It can be made of discretized sub-sources as long as they remain coherent and with the same directivity. We have considered here an array of connected waveguides covering the surface of a glass substrate. These waveguides spread the laser light out on the whole surface. Discretized plasmonic nanoantennas positioned at specific locations along the waveguides realise then the out-of-plane coherent beam steering.

Directive light emission has been demonstrated in Yagi Uda antenna based integrated structures [1-3]. In all cases, the most part of the far-field radiation is directed toward the higher refractive index medium, with an angle determined by the refractive index contrast. So in these configurations, there is no way to chose the beaming angle, unless by changing the refractive index contrast.

Here, in order to obtain an out-of-plane beaming having a full angle control at visible wavelength, we propose an emitter structure composed of a plasmonic nanoantennas periodic grating coupled to a monomode dielectric $\mathrm{Si}_{3} \mathrm{~N}_{4}$ waveguide embedded in a homogenous $\mathrm{SiO}_{2}$ medium [4,5]. The Bragg grating function of each scatterer controls the directivity (direction and divergence) of the beam, whereas the localized plasmonic resonance of nanoantenna monitors the coupling efficiency (Fig.1).
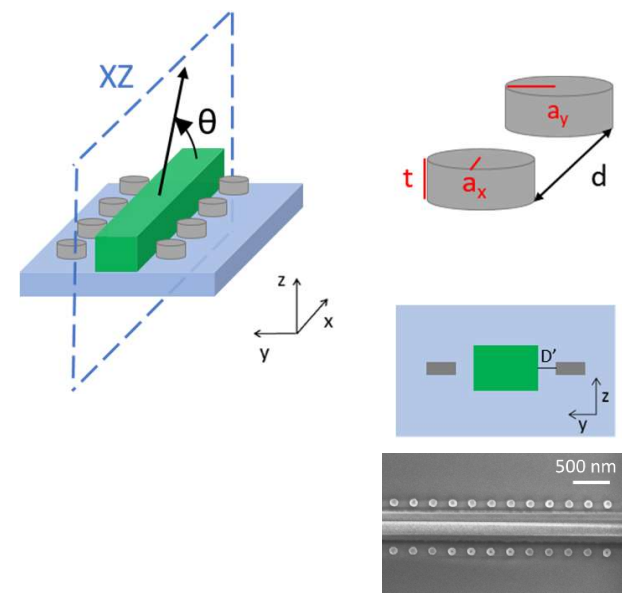

Fig. 1, elementary integrated scatterer made of two silver NP chain positioned at each side of the silicon nitride waveguide, and related design parameters $\left(a_{x}, a_{y}, d\right.$ and $\left.D^{\prime}\right)$. Corresponding fabricated device (SEM image)
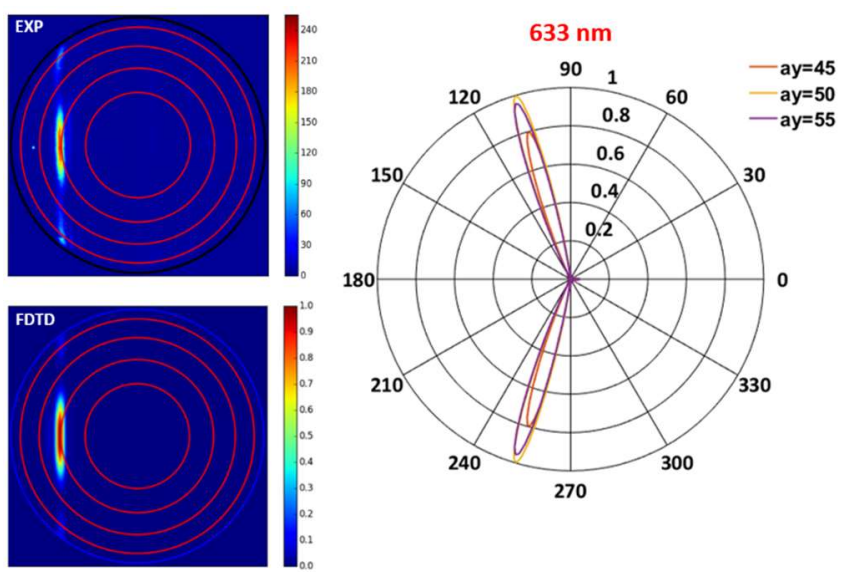

Fig. 2, (left) experimental and numerical far field map of emitted light à $633 \mathrm{~nm}$. (right) Tunability of the waveguide-nanoantenna coupling efficiency by NP size ( 3 values of the nanorod ellipse large diameter $\mathrm{a}_{\mathrm{y}}$ ) for $\mathrm{d}=300 \mathrm{~nm}$.

We show that each grating plays the role of a scatterer with full control of the beam shape and emitted power by independent design parameters (Fig. 2): up to 8 emitters can be positioned in series delivering similar emission diagram in shape and intensity. This confirms the feasibility of a large area coherent source by using plasmonic nanoantenna gratings positioned near a dielectric waveguide [6]. First experimental results validate the approach. Additionally we identify and analyse coupling mechanisms between nanoantenna chains and surface lattice modes, for the first time in a waveguiding excitation configuration.

\section{Example References}

[1] A. Curto et al., "Unidirectional Emission of a Quatum Dot Coupled to a Nanoantenna,' Science, vol. 329, p.312-315, 2010

[2] T. Kosako et al., "Directional control of light by a nano-optical Yagi-Uda antenna," Nature Photon., vol. 4, p. 312-315, 2010

[3] F. B. Arango et al., « Plasmonic Antennas Hybridized with Dielectric Waveguides, » ACS Nano, vol. 6, 10156-10167, 2012 .

[4] M. Février et al., "Giant coupling effect between metal nanoparticle chain and optical waveguide", Nanoletters, 12, p. 1032-1037, 2012.

[5] M. Février et al., "Metallic nanoparticle chains on dielectric waveguides: coupled and uncoupled situations compared", Optics Express, vol.21, No.21, 24504-245013, 2013.

[6] B. Leroy et al, "Integrated Nanoantenna Gratings For Planar Holographic Signalisation System ," Asia Communications and Photonics Conference (ACP) 2018, paper M2J.7, 26-29 October 2018, New Century Grand Hotel, Hangzhou, China 